\title{
La integridad académica: el dilema de la formación médica
}

\section{Academic integrity: The dilemma of medical education}

\section{A integridade acadêmica: o dilema da formação médica}

\author{
Ana Isabel Gómez Córdoba* \\ Boris Julián Pinto Bustamante
}

Fecha de recepción: 2 de octubre 2017

Fecha de evaluación: 2 de noviembre 2017

Fecha de aceptación: 5 de noviembre 2017

Fecha de publicacion en linea: 1 de diciembre de 2017

DOI: http://dx.doi.org/10.18359/reds.3248

\section{Cómo citar este artículo:}

Gómez Córdoba, A. I. y Pinto Bustamante, B. J. (2017). La integridad académica: el dilema de la formación médica. Revista Educación y Desarrollo Social, 11(2), 162-188. DOI: org/10/18359/reds.3248.

Médica cirujana, especialista en Pediatría, en Gerencia en Salud Pública y Derecho Médico Sanitario, Universidad del Rosario; magíster en Bioética, Universidad El Bosque; doctora en Ciencias Jurídicas, Pontificia Universidad Javeriana. Profesora titular, vicedecana de la Escuela de Medicina y Ciencias de la Salud, Universidad del Rosario, y codirectora de la Especialización en Derecho Médico Sanitario de la Universidad del Rosario, Bogotá, Colombia. Correo electrónico: anai.gomez@urosario.edu.co. ORCID: http://orcid.org/0000-0001-6328-917X.

** Médico cirujano, especialista y magíster en Bioética, Universidad El Bosque; candidato a doctorado en Bioética de la misma universidad. Profesor investigador de Bioética de la Universidad El Bosque, y profesor principal de carrera de la Escuela de Medicina y Ciencias de la Salud, Universidad del Rosario, Bogotá, Colombia. Correo electrónico: boris.pinto@urosario.edu.co. ORCID: http://orcid.org/0000-0002-2463-4214. 


\title{
Resumen
}

La integridad académica es uno de los propósitos principales de la educación de alta calidad. No obstante, el fenómeno de deshonestidad académica es un problema creciente a nivel global que se ha sofisticado por el acceso a las nuevas tecnologías de la información y la comunicación. Este trabajo aborda el problema de la deshonestidad académica en el contexto de la enseñanza de la medicina, sus causas, modalidades, factores predisponentes y estrategias de prevención, detección y sanción. La deshonestidad académica es un fenómeno multicausal en el que confluyen factores relativos al entorno y la cultura moral, factores personales, currículo oculto, políticas institucionales, así como modelos de enseñanza basados en los resultados académicos, más que en el proceso de aprendizaje colaborativo. Dentro de las prácticas deshonestas se pueden citar el plagio, la colusión, la falsificación, el engaño y la suplantación. La deshonestidad académica genera consecuencias para los estudiantes, los docentes, las instituciones, el sistema educativo, la profesión médica y la sociedad, pues es papel de las instituciones educativas no solo la producción de técnicos competentes, sino también de ciudadanos responsables. El presente trabajo propone algunas estrategias para enfrentar este problema, como la evaluación del desarrollo moral al ingreso a los programas académicos y su supervisión durante el proceso formativo, entre otras.

Palabras clave: ética, educación médica, plagio, valores sociales, profesionalismo.

\begin{abstract}
Academic integrity is one of the main purposes of high quality education. However, the phenomenon of academic dishonesty is a growing problem at the global level that has become more sophisticated due to new information and communication technologies. This paper addresses the issue of academic dishonesty in the context of teaching medicine, its causes, modalities, predisposing factors and strategies for prevention, detection, and punishment. Academic dishonesty is a multi-causal phenomenon in which factors related to the environment and moral culture, personal factors, hidden curriculum, institutional policies, as well as teaching models based on academic results, rather than the collaborative learning process. Within the dishonest practices, we can mention plagiarism, collusion, falsification, deception, and impersonation. Academic dishonesty generates consequences for students, teachers, institutions, the educational system, the medical profession, and society because it is the role of educational institutions not only the production of competent technicians but also responsible citizens. The present paper proposes some strategies to face this problem, such as the evaluation of moral development upon admission to academic programs and their supervision during the training process, among others.

Keywords: Ethics, medical education, plagiarism, social values, professionalism.
\end{abstract}

\section{Resumo}

A integridade acadêmica é um dos principais objetivos da educação de alta qualidade. No entanto, o fenômeno da desonestidade acadêmica é um problema crescente a nível global que se tornou mais sofisticado devido ao acesso às novas tecnologias da informação e a comunicação. Este artigo aborda o problema da desonestidade acadêmica no contexto do ensino da medicina, suas causas, modalidades, fatores predisponentes e estratégias de prevenção, detecção e punição. A desonestidade acadêmica é um fenômeno multicausal em que convergem os fatores relacionados ao ambiente e a cultura moral, fatores pessoais, currículo escondido, políticas institucionais, bem como modelos de ensino baseados nos resultados acadêmicos, mais do que no processo de aprendizagem colaborativa. Dentro das práticas desonestas podemos mencionar o plágio, a colusão, a falsificação, o engano e a usurpação. A desonestidade acadêmica gera consequências para os estudantes, os professores, as instituições, o sistema educacional, a profissão médica e a sociedade, pois é papel das instituições educacionais não apenas a produção de técnicos competentes, mas também de cidadãos responsáveis. O presente artigo propõe algumas estratégias para enfrentar este problema, como a avaliação do desenvolvimento moral após a admissão aos programas acadêmicos e sua supervisão durante o processo de formação, entre outras.

Palavras-chave: ética, educação médica, plágio, valores, sociais, profissionalismo. 


\section{Casos}

1. Ana María considera que no se encuentra lo suficientemente preparada para presentar un examen que determinará el promedio final de una asignatura. Decide presentar una incapacidad falsa que le ayuda a ser evaluada por fuera de los tiempos estipulados y así contar con mayor tiempo para estudiar.

2. Juan Carlos permite que su compañero Martín copie sus respuestas durante el examen. Él lo quiere ayudar, debido a que si no obtiene una nota alta en esta evaluación, pone en riesgo la beca que tiene.

3. En el grupo de estudio de Fernando, cuatro de sus compañeros desarrollan de manera incompleta las actividades que les son asignadas; esta situación afecta el desempeño del equipo. Cuando este se queja, sus compañeros lo tildan de "sapo", de "soplón".

4. Manuel se encuentra en un grupo que cuenta con personas que tienen un muy buen rendimiento académico. Esto le permite pasar "raspando" las asignaturas, gracias a que el cómputo final, entre las evaluaciones en grupo y la individual, le permite alcanzar la nota mínima requerida para aprobar.

5. Esteban siente rabia e impotencia por el comportamiento de algunos de sus compañeros de semestre que copiando logran mejores notas que las que él obtiene estudiando. Considera que esta situación es injusta, más aun cuando el acceso a becas está condicionado por un buen desempeño académico. Desearía reportar esta situación, pero teme las consecuencias que esto acarrearía en la relación con sus compañeros. Le duele que las directivas del programa y sus docentes no hagan nada al respecto.

6. Manuel asiste a la reunión de diez años de egresados de su programa de Medicina. Durante el encuentro, algunos de sus compañeros le recuerdan, entre bromas, su habilidad para copiar y lo cuestionan sobre su actual desempeño profesional.

7. María, docente de programa de Medicina, detecta plagio en un trabajo mediante un software. Se pregunta cómo debe manejar esta situación: si debe asignar cero o iniciar un proceso disciplinario.

8. Carlos, médico general egresado de un prestigioso programa de Medicina, enfrenta una demanda penal y una queja ante el Tribunal de Ética debido al daño producido en un paciente, por su falta de experticia profesional. En la facultad, durante su proceso de formación, sus compañeros lo reconocían por su conducta académica deshonesta.

9. Paula emplea su conocimiento en sistemas de información y diversos dispositivos para copiar durante sus exámenes. Tiene uno de los 
mejores promedios de su semestre. No siente culpa o remordimiento respecto a esta práctica, ni cree que vaya a afectar su futuro desempeño profesional.

10. Mercedes, docente de una facultad de Medicina, al ser cuestionada sobre la magnitud del fenómeno de falta de integridad académica de sus estudiantes, considera que a lo sumo de un 5 a un $10 \%$ de ellos lo hace, y que posiblemente cuando se gradúen será la misma sociedad la encargada de limitar su ejercicio profesional.

11. Un estudiante se encuentra de turno con su instructor. Durante la noche es testigo de cómo este se ausenta del servicio con otro de los profesionales del equipo de salud y le entrega su clave del sistema de información del hospital para que coloque las notas en su nombre.

12. Gabriel está en su año de internado y debe examinar a 20 pacientes durante su ronda nocturna. Al día siguiente es llamado por el jefe de un servicio para que explique por qué coloco en la nota de la historia clínica: "Fondo de ojo normal", en un paciente con una catarata congénita.

13. Francisco comenta a sus compañeros los tópicos y preguntas de la Evaluación Clínica Objetiva Estructurada (ECOE) que acaba de presentar, antes de que ellos ingresen a la sala de examinación.
14. El grupo de Mariana estudia para los exámenes únicamente con los bancos de preguntas que se han ido pasando de generación en generación en su Facultad de Medicina. ¿Para qué esforzarse si ellos saben que un alto porcentaje de estas preguntas vuelven a repetirse?

15. Un estudiante de Medicina se encuentra rotando por cirugía. Asiste como ayudante a un procedimiento quirúrgico en el cual se presenta una complicación. Posteriormente, este caso es llevado a la reunión del servicio y cuando el residente lo presenta la descripción no corresponde a la realidad de los hechos ocurridos.

16. Un estudiante, ante solicitud de su profesor de presentar el análisis de un artículo de investigación como parte de las tareas de su asignatura, decide presentar un trabajo previamente realizado por él en un semestre anterior, el cual ya había sido evaluado y calificado. No advierte de este hecho a su profesor.

\section{Introducción}

Como docentes de las áreas de bioética y bioderecho de un programa de Medicina, es motivo de preocupación y desencanto observar la práctica recurrente del engaño en algunos estudiantes durante su proceso de formación. Esto se manifiesta, algunas veces, como un conjunto de prácticas deshonestas: por ejemplo, la copia, el fraude, el plagio o 
la fabricación de los datos clínicos de un paciente cuando presentan un caso en la revista clínica, y otras, en acciones que se encuentran en una zona gris de la ética, como son el ausentismo y la elección de la vía del menor esfuerzo para lograr graduarse.

Esta preocupación tiene su razón de ser, no solo en las dudas que se ciernen sobre la adquisición de las competencias médicas del egresado, y cuya garantía es responsabilidad de la universidad y del programa de Medicina, sino también por el efecto que este comportamiento reviste para la profesión en su conjunto.

La medicina, el derecho y el sacerdocio son las tres profesiones educadas, originales de las universidades medievales europeas las cuales, como "profesiones prototípicas de la sociedad antigua" (Lázaro y Gracia, 2006), se diferenciaban de las denominadas profesiones ocupacionales. Tradicionalmente, las profesiones educadas se caracterizaban por su importancia para la sociedad, las "ideologías del conocimiento y la experiencia especiales", su "probidad moral" y la existencia de instituciones de "capacitación y credencialización". Todo esto les permite ostentar un estatus privilegiado que se traduce en ventajas sociales, económicas y políticas, así como la protección del Estado en el mercado laboral (Freidson, 2001).

Freidson (1988), en su obra La profesión médica: un estudio de sociología del conocimiento aplicado, señala que la medicina es una práctica que goza de autonomía profesional para determinar los contenidos y límites del ejercicio profesional, y que ha logrado el derecho al control de su práctica y organización del trabajo, lo que le otorga el poder dentro de la sociedad en relación con el proceso salud-enfermedad, así como las técnicas para intervenirlo. La medicina es entonces una profesión con autonomía, y el Estado le permite "el control sobre los aspectos técnicos de su trabajo, y sobre la organización social y económica del mismo", así como determinar quién legítimamente puede ejercerla (Rodríguez, 1981, p. 143). En este orden de ideas, la sociedad espera que un profesional de la medicina posea el conjunto de conocimientos, destrezas y actitudes para resolver las problemáticas de salud de las personas y las comunidades, que anteponga el mejor interés de sus pacientes a su propio interés, y que demuestre capacidad para autorregularse, dado el poder y los privilegios que se le otorgan. Estas virtudes son difícilmente alcanzables por una persona que ha fundado su proceso de formación en el engaño, a sí mismo y a los demás. No es de extrañar la desconfianza creciente de la sociedad en los profesionales de la medicina y las restricciones que mediante instrumentos jurídicos y administrativos ha impuesto al ejercicio de esta; podría decirse que son más libres los jueces al tutelar el derecho a la salud y ordenar la práctica de un procedimiento, que los médicos en sus consultorios.

Para las universidades, docentes y directivos la falta de honestidad académica 
es el mayor fracaso pedagógico, más aún cuando los programas de Medicina de vanguardia se fundamentan en el aprendizaje autónomo de los estudiantes, la adecuada interacción del trabajo en equipo, el aprendizaje para la comprensión y la evaluación formativa.

A manera de ejemplo, el programa de Medicina de la Universidad del Rosario tiene un plan de estudios fundamentado en Resultados del Aprendizaje Esperados que articulan verticalmente el currículo, así como Actividades Integradoras de Aprendizaje por Sistemas (AIAS), en las cuales se integran las ciencias básicas, biomédicas, clínicas, sociohumanísticas y de salud de poblaciones. En estas, grupos pequeños de estudiantes resuelven los problemas relacionados con el proceso salud-enfermedad de las personas y las poblaciones mediante el aprendizaje basado en la comprensión (EpC) y el trabajo en equipo. Cada estudiante es responsable de su propio aprendizaje y el de sus pares, y el docente tiene un rol de facilitador. Además, se fomenta el contacto temprano con los pacientes (Quintero, 2014).

El programa de Medicina se apoya en una plataforma tecnológica que permite el acceso en línea al material pedagógico, a los e-portafolios y la posibilidad de realizar pruebas desde lugares remotos. La evaluación es un instrumento pedagógico y tiene un carácter sumativo y formativo. Debido a que el programa busca el aprendizaje para la comprensión, y no el memorístico, tiene un número menor de créditos, mayor flexibilidad y tres tipos de profundizaciones. El estudiante tiene entonces la posibilidad de elegir de forma autónoma la exploración de temáticas adicionales a las determinadas por el docente, con espacios de práctica dispuestos para tal fin. Se cuenta también con el apoyo de un grupo de mentores (Quintero, 2014).

En este plan de estudios el estudiante debe desarrollar la capacidad de liderar su propio aprendizaje, de tal forma que adquiera una serie de competencias, así como la capacidad de seguir aprendiendo a lo largo de su vida. En este entorno pedagógico resulta poco estimulante, e incluso molesto e injusto para el estudiante que es capaz de responder de forma autónoma por las actividades que le son encomendadas dentro del trabajo en equipo, ver que aquellos que copian o eligen la línea del menor esfuerzo son "arrastrados" por su esfuerzo, como rémoras que se alimentan del trabajo de un gran pez o parásitos que viven a expensas de su huésped. En un espacio donde se brindan oportunidades permanentes para volver a ser evaluado hasta que se garantice el resultado esperado de aprendizaje, no es fácil comprender el hecho de que se opte por el engaño. Puede ser contraproducente el contacto temprano con los pacientes, cuando quien lo hace, además de ser inexperto, es un tramposo.

Nos preguntamos entonces si nuestra comunidad académica es reflejo de las enfermedades que aquejan a nuestra sociedad, en la que impera la cultura del más vivo, caracterizada por la corrupción 
de unos y el desdén, la apatía y hastío de otros, donde cada persona procura sobreaguar y sobrevivir individualmente al siguiente día, y donde se estigmatiza a quien se atreve a revelar o enfrentar estas prácticas. Se califica incluso despectivamente a quien denuncia y se le denomina "sapo", "delator", "soplón", "traidor", "espía”, "bocón”, o "lambón”.

Por todas estas razones, consideramos que una de las mayores encrucijadas morales de los estudiantes, que se extiende desde la formación de pregrado hasta las instancias de postgrado, es la de iengañar o no engañar?, y que esta, incluso, trasciende a su vida profesional y personal. Pero, ¿cuál es la solución a este dilema?, ¿seleccionar estudiantes proclives a la virtud? ¿Cómo se podría implementar tal proceso de selección?, ¿solicitar a los colegios certificados de buena conducta para los aspirantes?, ¿volver a los currículos antiguos en los cuales más del $40 \%$ de los estudiantes desertaban? ¿Convertirnos en universidades policiales, que como un "Gran Hermano" vigilan los movimientos de cada uno de los miembros de su comunidad y sancionan de manera ejemplar a sus estudiantes dentro de una política de cero tolerancia?, o ¿dejar que la sociedad posteriormente se encargue de seleccionar a estos médicos, y que sean los pacientes y la profesión en su conjunto los que paguen el costo de la incapacidad de los egresados para autorregularse?

Con estos interrogantes en mente reflexionaremos a continuación sobre el tema de la falta de integridad académi- ca en la educación médica y sobre las posibles soluciones a este problema, con el fin de identificar estrategias que promuevan un entorno favorable para una cultura de la integridad académica.

Para cumplir con este objetivo se incluirán las siguientes temáticas: definiciones sobre el concepto de deshonestidad académica, las formas desde las que se presenta en la educación médica de pregrado, su frecuencia y factores que la refuerzan o la evitan; las modalidades que se emplean para faltar a la integridad académica, el efecto de este tipo de prácticas y las medidas para contrarrestarlas. También se comentará brevemente cómo es el razonamiento moral de adolescentes y adultos jóvenes y su incidencia en el aprendizaje autónomo y la autorregulación.

\section{El concepto de deshonestidad académica o falta a la integridad académica}

La deshonestidad académica se puede definir como un "acto intencional de fraude, en el cual el estudiante reclama para sí el crédito por el esfuerzo o trabajo de otro sin su permiso, o usa materiales o produce información, sin autorización, en cualquier ejercicio académico" (Gehring y Pavela, 1994, p. 5). Incluye asistir a otros en actos de falta de honestidad, estorbar el trabajo académico de terceros (Gehring y Pavela, 1994), el engaño en el conocimiento, en el trabajo producido o en el desempeño de competencias de un estudiante en el espacio educativo (Rujoiu y Rujoiu, 2014). 
Los actos de engaño que de manera reiterativa se describen en la literatura, así como en los códigos de conducta de las universidades, son el plagio, la colusión, la falsificación, el engaño y la personificación o suplantación. A manera de ejemplo, emplearemos las definiciones que aporta la Universidad de Edimburgo (2016) de estos conceptos:

- Plagio: "presentación del trabajo de otra persona como propio sin poner en conocimiento la fuente o sin el permiso del creador, intencional o no intencionalmente".

- Colusión: "es una forma de plagio donde se da la colaboración de un estudiante en un trabajo no autorizada o no atribuida".

- Falsificación: es "la tentativa de presentar datos, evidencia, referencia, citación o resultados de experimentos, ficticios o distorsionados".

- Copia: "intento de obtener o dar asistencia en una evaluación o trabajo sin haber reconocido que este incluye presentar trabajo que no es propio".

- Engaño: "es la falta de honestidad que busca ventaja, como es el uso del propio trabajo previamente hecho".

- Personificación o suplantación: "asumir la identidad de otra persona con la intención de recibir o ganar una ventaja injusta".
No obstante, debe aclararse que el significado de deshonesto es distinto según la cultura, la institución, o el programa, de ahí que un primer propósito, de cualquier esfuerzo para crear una cultura de integridad académica, debe apuntar a clarificar este concepto y las acciones que incluye, de manera explícita y, si es posible, con ejemplos, para así poder socializarlo en la comunidad académica (Bates, Davies, Murphy y Bone, 2005).

Las faltas a la integridad académica pueden tener un carácter individual o colectivo, tradicional o moderno, simple o ingenioso, fácil o laborioso. Algunas de las prácticas que están incluidas dentro del concepto de deshonestidad académica son las siguientes (Rujoiu y Rujoiu, 2014):

- Mentir sobre circunstancias médicas o de otro tipo para obtener un trato preferencial o mayor tiempo para presentar trabajos o evaluaciones. Incluye fingir estar enfermo o enfrentar una fuerza mayor o un caso fortuito para justificar la ausencia a actividades académicas.

- Usar en los trabajos material de libros, artículos o páginas web sin otorgar el reconocimiento al autor, mediante la cita bibliográfica. o parafrasear sin el empleo de la cita bibliográfica.

- Emplear trabajos previamente presentados por estudiantes que han adelantado sus estudios con anterioridad. 
- Acceder o sustraer exámenes, bien sea de los computadores de los docentes o en el momento de su impresión.

- Falsificar documentos para obtener beneficios, como es el caso de las incapacidades o notas.

- Ayudar a otro estudiante a realizar tareas o trabajos que han sido diseñados para ser completados de forma individual.

- Omitir resultados no deseados en un experimento o manipular los datos.

- Encubrir una falta a la integridad académica de uno de sus pares.

- No asistir a clase y pedir a un compañero que firme en su lugar.

- Suplantar a otro estudiante en cualquier actividad académica.

- Entregar un trabajo por el cual ya se había recibido una nota.

Se incluye además el copiar durante los exámenes, de otros compañeros, o con el uso de distintos tipos de dispositivos móviles, inalámbricos o material físico no autorizado; mentir durante el proceso de examinación de un paciente y falsificar los datos de los antecedentes o datos del examen físico o los resultados de las pruebas realizadas; compartir información sobre la ECOE (Kusnoor

170 y Falik, 2013). También se incluyen diversas formas de corrupción: pago por el acceso a evaluaciones, por el cambio en las notas o por la realización por parte de un tercero de trabajos escritos (Bjorklund y Wenestam, 1999). En la zona gris están prácticas como el estudio de pruebas de años anteriores, el ausentismo o el cumplimiento únicamente de los requisitos mínimos.

Algunos estudios afirman un distinto grado de reconocimiento por parte de los estudiantes de este tipo de prácticas como conductas que afectan la integridad académica. Así es como la mentira, la copia y el robo son fácilmente identificados como incorrectos, lo que no ocurre con las diversas formas del plagio. Lamentablemente, si bien se identifica que una conducta es incorrecta, esto no impide que finalmente se incurra en ella (Ghias, Lakho, Asim, Azam y Saeed, 2014).

Si bien nuestro objetivo se concentra en las faltas a la integridad académica, existe otro tipo de conductas que se dan dentro y fuera de las universidades, que son motivo de preocupación y que nos cuestionan sobre si el estudiante involucrado debería recibir el grado como médico. Algunas de estas son el robo, la violencia física o sexual, el abuso de alcohol o sustancias psicoactivas, el terrorismo, la falsificación, la posesión de pornografía infantil, entre otros (Bonke, 2006).

\section{Frecuencia de la falta de honestidad académica}

Son múltiples los artículos que abordan la falta de honestidad en el proceso de formación médica en el mundo. Estos pueden ser encontrados con diversas denominaciones: práctica antiética, fraude 
o mala conducta académica (Rujoiu y Rujoiu, 2014). Todos ellos apuntan a un aumento de la frecuencia de estas prácticas en el proceso de educación médica, a pesar de que en los programas se cuente con formación ética a lo largo del plan de estudios (Rennie y Rudland, 2003).

La frecuencia con la que se presenta este fenómeno es variable y depende de diferentes factores, como el país, el carácter público o privado de la institución educativa, el género, el periodo de estudio (preclínico o clínico) (Ghias et al., 2014). También intervienen factores como el tipo de personalidad y el trasfondo cultural (Vengoechea, Moreno y Ruíz, 2008).

En general, algunos estudios afirman que las mujeres son menos proclives a la deshonestidad académica (Rujoiu y Rujoiu, 2014). Las faltas a la integridad académica tienen una aceptación social mayor en el periodo clínico que en el preclínico; esto se debe a una desensibilización progresiva durante el proceso de formación médica (Rennie y Rudland, 2003).

En España, una investigación encontró que el 59,6\% de un grupo de 250 estudiantes universitarios admitieron haber recurrido al engaño alguna vez durante un examen, y el 31,6\% en un trabajo escrito (García y Manchado, 1998). En la India, un reporte encontró un 88\% de casos de fraude académico (Sheriff, Sheriff y Manopriya, 2000). En Estados Unidos, un $39 \%$ de los estudiantes ha observado este comportamiento entre sus pares (Baldwin, Daugherty, Rowley y Schwarz et al., 1996). En Pakistán, el $55 \%$ de los estudiantes admite haber engañado al menos una vez durante su proceso de formación (Hafeez, Khan, Jawaid y Haroon et al., 2013). En Nueva Zelandia, se reporta en un estudio que el $91 \%$ de los estudiantes copió, 34\% engañó y 60 \% cometió colisión (Henning, Ram, Malpas, Shulruf, et al., 2013). En Colombia, el 94,4\% de un grupo de estudiantes admitió haber cometido fraude durante su carrera académica (Ordoñez, Mejía y Castellanos, 2006).

Debido a que se trata de un fenómeno oculto, es difícil establecer la magnitud real de su presentación. No obstante, más allá de la cifra, debe recordarse que parte del problema tiene su origen en desestimar su prevalencia, así como las consecuencias de no intervenirlo.

\section{¿El engaño se ha sofisticado?}

Las formas tradicionales de engaño incluyen prácticas deshonestas como el acceso a bancos de preguntas, la compra de exámenes o copiar de un compañero durante la evaluación.

Los docentes han dado respuesta, en la medida de sus posibilidades, a estas prácticas deshonestas: se cambia el orden de las preguntas de una evaluación de manera aleatoria, se imprime el examen por fuera de la universidad, se dividen los grupos y se aumenta progresivamente el número de personas que observan el momento de la evaluación (aunque no siempre es fácil contar con personas comprometidas para apoyar a un docente 
que tiene a su cargo más de 100 estudiantes y que efectivamente estén atentos a la copia), o prefieren hacer evaluaciones orales a pesar de lo dispendioso que esto resulta. Las instituciones, por su parte, han desarrollado reglamentos académicos en los cuales estas prácticas son sancionadas y comités disciplinarios revisan las denuncias y aplican las sanciones del caso.

Sin embargo, con el acceso a nuevas tecnologías de la información y la comunicación, que hacen parte de nuestra vida diaria o que soportan los procesos formativos, las faltas a la integridad académica han adquirido nuevos matices (Pupovac, Bilic-Zulle y Petrovecki, 2008): ya no se recurre exclusivamente a los apuntes o al libro en físico, sino a textos en formato electrónico que son asequibles a través de las pantallas táctiles de los celulares o iPads. Los computadores donde se almacenan las pruebas son hackeados; además, se ingresa, sin autorización, a los recursos pedagógicos que reposan en las plataformas tecnológicas durante los momentos de evaluación, o simplemente se finge contestar una prueba, pero se deja la evaluación abierta para ser completada posteriormente dentro de los tiempos para la evaluación, sin ninguna supervisión y con el soporte de todo tipo de recursos.

En el caso de trabajos escritos, se copia y pega del internet, sin el adecuado uso de la citación bibliográfica, con el consecuente plagio a la propiedad intelectual de otro.
También se reporta el uso de dispositivos electrónicos como celulares para acceder a chats de conversación con personas que se encuentran fuera del salón de evaluación, o se crean grupo de Whatsapp donde todos aportan para la respuesta de la evaluación o se comparten fotos del examen que ya ha sido contestado. Además, existen relojes que cumplen con estos mismos fines, para no hablar de las gafas de Google, que al menos son fácilmente detectables. Los estudiantes que finalizan primeros la prueba se comunican con los que aún no la han hecho para enviarles las respuestas. Si bien se prohíbe el ingreso y uso de celulares y otro tipo de dispositivos, estos se ocultan, a sabiendas de que lo único que le falta hacer a las universidades es catear a los estudiantes o colocar detectores de metales. Incluso se emplean dispositivos auditivos a través de los cuales se puede mantener comunicación con el exterior, donde una persona puede dictar las respuestas en un examen oral (González, 2014).

Paralelamente, las instituciones educativas, han sofisticado sus prácticas. A manera de ejemplo se emplean programas de software que permiten detectar rápidamente el plagio. En este caso, «WebCT, Blackboard proporciona la herramienta SafeAssign, que entrega al profesor un informe con el porcentaje de texto extraído de otras fuentes» (Aunión, 2013). También está Turnitin, un software empleado para detectar plagio (Batane, 2010). 
Algunas universidades europeas han optado por colocar inhibidores de frecuencia de extensión limitada al aula durante sus exámenes, de tal forma que bloquen las transmisiones de celulares o internet (Aunión, 2013), aunque algunos reportan que esta medida es inviable y poco efectiva. Otros recurren al uso de aplicaciones que detectan celulares, como es el caso de pocket hound (González, 2014).

Finalmente, se están empleando cámaras que graban el momento de la evaluación, como soporteen los procesos disciplinarios. Parecería que lo único que nos falta es que sobrevuelen drones durante los exámenes.

\section{Factores que propician o evitan la falta de integridad académica}

Las faltas a la integridad académica tienen un origen multicausal y son de carácter interno y externo. Algunas de ellas son:

- Antecedentes de copia en el colegio (Anderson y Obenshain, 1994).

- Percepción moral al ingreso a la facultad de medicina (Vengoechea et al., 2008).

- Capacidad para identificar y resolver dilemas éticos (Henning et al., 2013).

- Momento de la formación: los estudiantes en fase clínica tienen mayor dificultad en identificar comportamientos antiéticos que los que se encuentran en la fase básica y biomédica (Kulac, Sezik, Asci y Doguc, 2013).
- Características de la personalidad: estudiantes con baja autoestima, comportamiento neurótico o ambiciones desproporcionadas (Anderson y Obenshain, 1994).

- Conciencia de mejor rendimiento de los pares y experiencias previas de falla académica, en adición a la dificultad e importancia de la prueba (Baird, 1980). Aunque, debe señalarse que sorprendentemente no solo copian los estudiantes con regular rendimiento académico, sino que también lo hacen aquellos que tienen excelentes notas (Smith, 2000).

- Características del sistema de formación médica que producen estrés y síndrome de agotamiento en el estudiante: alto nivel de exigencia y sobrecarga académica, con incentivos fundamentados en la excelencia; a esto se suman los altos costos de las matrículas y los elevados índices de deserción (Dyrbye, Massie, Eacker et al., 2010).

- Aceptación social de las faltas a la integridad académica y avance en la carrera. Los infractores consideran que esta conducta es transitoria y no afectará su desempeño profesional futuro. La conducta de los pares es uno de los factores más importantes para prevenir o propiciar la falta a la integridad académica (McCabe y Trevino, 2001).

- Fallas en la prevención, detección y sanción: la frecuencia de faltas a la integridad es alta y su detección baja, 
así que, si es fácil engañar, ipor qué no hacerlo? (Davis y Ludvigson, 1995).

- Grupos mayores de estudiantes que inciden en la falta de personalización del proceso formativo y la menor posibilidad de control (Ledesma, 2011).

- Temor de los estudiantes a rechazar o denunciar este tipo de prácticas, por posible matoneo por parte de sus compañeros (Rennie y Crosby, 2002). Por esta razón, se prefiere la denuncia anónima o la gestión informal de este tipo de conductas (Newstead, Franklyn-Stokes y Armstead, 1996).

- La existencia de un currículo oculto, en el cual las faltas a la integridad académica están presentes en el entorno educativo (Christakis y Feudtner, 1993).

- Tipo de evaluación: las pruebas de selección múltiple favorecen la copia (Davis y Ludvigson, 1995).

- Aversión por el docente o la asignatura (Davis y Ludvigson, 1995).

- Entendimiento de la aceptación de la copia como una forma de expresar amistad y solidaridad con un par (Franklyn-Stokes y Newstead, 1995).

Algunas de las razones aducidas por los estudiantes que no faltan a la integridad académica para soportar su conducta son

174 el reconocimiento del carácter inmoral y deshonesto de la acción, la cual se constituye en una práctica injusta con otros estudiantes; la falta de conoci- miento o experiencia para hacerlo y el miedo al castigo y la vergüenza en caso de ser detectado. Otros consideran que es innecesario, afecta su orgullo personal y devalúa la legitimidad de los logros (Bjorklund y Wenestam, 1999).

Aquellos estudiantes que optan por no faltar a la integridad académica tienen un comportamiento que es calificado por algunos autores, incluso, como heroico, debido a que tienen características personales como el coraje, la capacidad de empatía, la honestidad. (Staats, Hupp, Wallace y Gresley, 2009). Debe señalarse en este punto que uno de los más poderosos factores que incentivan negativamente la falta de integridad académica es que esta práctica sea desaprobada por los pares (McCabe y Trevino, 1997).

En este orden de ideas, es importante contar con estrategias que permitan identificar los factores contributivos a las faltas a la integridad académica e intervenirlos, así como propiciar aquellos que actúan como barreras de contención (Henning et al., 2013).

\section{¿Existe tolerancia por parte de las universidades a las faltas a la integridad científica?}

¿Cómo responden las universidades y los docentes a las faltas a la integridad académica? Estudios en otros contextos sobre el tema han revelado que solo el $44 \%$ de los docentes que detectan faltas a la integridad académica las denuncia (Scanlan, 2006). Este fenómeno se puede explicar, bien porque existe una cultura no punitiva que busca aprender del error, 
o porque existe una velada tolerancia a esta clase de hechos.

Previamente, se señaló la existencia de un currículo oculto, especialmente en los momentos de encuentro con el paciente en el ámbito clínico, donde el estudiante es expuesto a desviaciones a los códigos de ética profesional, a una estructura jerarquizada, donde él es la base de la pirámide de poder y a lo que algunos denominan modelos de enseñanza por humillación (Bermeo, Castaño-Castrillón, López-Román, Téllezy Toro-Chica, 2016). Esto hace que, en la medida en la que se avanza en la carrera y aumenta la práctica clínica, el comportamiento del estudiante sea más cínico, se muestre emocionalmente distante del paciente y menos empático (Neumann, Edelhäuser y Tauschel et al., 2011). Se describe, además, un retroceso en el razonamiento moral a lo largo de los años, contrario a lo que ocurre en el proceso de formación de otras profesiones (Hren, Marušić y Marušić, 2011).

Algunos autores argumentan que la aparente tolerancia de docentes y directivos a los actos deshonestos tiene esta misma razón de ser. También están las barreras para el reporte de este tipo de conductas, como el miedo a las retaliaciones y las dificultades para mantener el anonimato (De Oliveira Vidal, Silva, Santos et al., 2015). También los grupos de estudiantes admitidos son cada vez mayores, sin que necesariamente se incremente de forma equivalente la planta docente; esto, sumado a las nuevas formas de engaño, dificulta la detección de las faltas a la integridad académica.
Los docentes no denuncian por la insuficiente evidencia de los hechos, por la ansiedad y el estrés asociados, así como por lo dispendiosos que resultan estos procesos (Staats et al., 2009). Debido a la juridificación de diversos ámbitos de la vida, incluida la relación estudiante-docente-universidad, se teme sancionar, por las dificultades en la comprobación de este tipo de prácticas (Hren et al., 2011).

Otras motivaciones para no intervenir en estas situaciones son: se tiene la creencia de que no se contará con el suficiente respaldo institucional; que este tipo de acciones policivas no hacen parte del rol docente; se lamenta dañar el futuro de una persona joven; se temen las retaliaciones jurídicas o las evaluaciones que los estudiantes hacen de los docentes, o se recuerdan episodios cuando el docente, durante su proceso de formación, tuvo similar comportamiento (Staats et al., 2009).

Finalmente, podríamos indicar que existe una corriente que considera que los reglamentos para los estudiantes deben propiciar su buen comportamiento autónomo y propender al aprendizaje a partir de las faltas, ponderando la pena de acuerdo con el momento de la vida académica en que se encuentra el estudiante. Así es como, durante el inicio del proceso de formación, es posible asignar una pena menos severa y con mayor carácter formativo.

A pesar de todos estos argumentos, ¿qué mensaje enviamos a los estudiantes que observan a sus compañeros faltar de las 
más diversas formas con su deber de honestidad, sin que nada les pase?, ¿no valdría la pena que valoremos, no solo la excelencia académica, sino también la forma como esta se logra?, ¿los mejores promedios siempre son los más honestos?, ¿deberíamos cambiar los sistemas de evaluación y de incentivos y visibilizar aspectos como la integridad, el altruismo, la capacidad de servicio?

\section{¿Los adolescentes están preparadospara el aprendizaje autónomo?}

Las tendencias pedagógicas en medicina se inclinan por el aprendizaje autónomo y el desarrollo del razonamiento moral que conduzca, entre otros efectos, a un maduro ejercicio de la autonomía y de la autorregulación profesional. Se entiende por aprendizaje autónomo aquel que se caracteriza por la personalización, la autodirección y la menor dependencia del docente, lo cual permite construir capacidades para el trabajo colaborativo en el espacio laboral (MacDougall, 2008).

El razonamiento moral favorece la distinción entre lo correcto y lo incorrecto, lo bueno y lo malo, lo justo y lo injusto. Esta es una competencia necesaria, no solo para la práctica de la medicina, sino también para la vida en sociedad como ciudadano.

Son distintas las teorías alrededor del concepto del desarrollo moral; una de las más relevantes (aunque no exenta de controversia) es la propuesta de Lawren- ce Kohlberg. Según este autor, el juicio moral evoluciona a través de tres etapas: preconvencional, convencional y posconvencional. En la etapa de la moralidad preconvencional, "la percepción del bien y del mal está vinculada a las consecuencias físicas beneficiosas o dañinas de las acciones del sujeto"; en la moralidad convencional "el sujeto vincula la bondad o maldad de sus actos a la adhesión a una regla o norma, aunque sin efectuar un juicio crítico sobre ella"; finalmente, en la moralidad posconvencional "la libre convicción del sujeto lo lleva a ajustarse a una norma a la cual se adhiere por íntima convicción". En general, el estadio de razonamiento moral se ve determinado por "los ámbitos culturales y sistemas de aprendizaje" (Delgado-Marroquín Altisent, Buil, Muñoz et al., 2014).

En este orden de ideas, ¿requiere el aprendizaje autónomo un determinado grado de desarrollo moral de los estudiantes?, ¿este puede ser evaluado en los aspirantes o durante el proceso de formación y al egreso?, ise deben personalizar las estrategias de aprendizaje acorde con el desarrollo moral de cada estudiante o debemos seleccionar para el ingreso a las escuelas y Facultades de Medicina aquellos que sean proclives a la autonomía y el autocontrol?

Efectivamente, el desarrollo moral puede ser evaluado y son diversos los instrumentos empleados, con diferente grado de eficacia. Pérez-Olmos y Dussán-Buitrago hacen una revisión de estas herramientas dentro de las que se incluyen la 
entrevista del juicio moral de Kohlberg y sus versiones modificadas, como la escala de reflexión socio-moral de Gibbs, el test de competencia moral de Rest, y la prueba Erasmo. Algunas escalas miden el juicio moral específicamente en médicos: la prueba de Hebert, Meslim y Dunn, la prueba Defining Issues Test (DIT) y Cuestionario de Opinión sobre Problemas Sociales (COPS) (Pérez-Olmos y Dussán-Buitrago, 2009). En este orden de ideas, se podría evaluar el razonamiento moral al ingreso, durante y al finalizar el proceso de formación, e implementar procesos individualizados que aseguren la adquisición del comportamiento autónomo y autorregulado requerido para ejercer la profesión (Patenaude, Niyonsenga y Fafard, 2003).

El estadio de razonamiento moral se ve determinado por los "ámbitos culturales y sistemas de aprendizaje". En general, la literatura sugiere que los puntajes de desarrollo del juicio moral de los estudiantes que ingresan a las facultades de Medicina son más altos en comparación con los de otras disciplinas. Sin embargo, como se señaló anteriormente, a lo largo del proceso formativo se hace evidente un retroceso, contrario a lo que sucede en otras profesiones (Delgado-Marroquín et al., 2014).

En los periodos de formación básica, los estudiantes de medicina son más proclives a orientar sus decisiones y argumentaciones en el contexto de la ley y del contrato implícito entre la profesión y la sociedad; posteriormente, se tornan más relativistas y basan sus juicios en las relaciones interpersonales, con lo cual se pierde la perspectiva social (Patenaude, Niyonsenga y Fafard, 2003).

Una investigación publicada en 2010, que tenía como objetivo caracterizar el razonamiento moral en estudiantes de Medicina de una universidad colombiana, con base en la escala de Kohlberg, encontró que "el razonamiento no se manifiesta como una línea de progreso, sino que evidencia rutas particulares e itinerarios diversos" (Escobar-Melo, Díaz, Páramo, Suárez y León, 2010). Además, Suárez y Díaz alertan sobre el hecho de que "el razonamiento bioético no parece tener relación con la asignatura formal de la carrera de medicina y sí más con la experiencia particular respecto del semestre o con la cohorte etaria" (Escobar-Melo et al., 2010, p. 53).

Estos hallazgos nos hacen cuestionar profundamente los procesos de formación profesional, específicamente frente a la adquisición de competencias ético-profesionales. Más allá de revisar contenidos de la bioética clínica y las responsabilidades éticas y jurídicas asociadas con el acto médico, se debe propender al desarrollar la sensibilidad y el juicio racional orientado hacia el bien. Los estudios señalan "que no se trata de la formación en ética, sino de la participación activa en la toma de decisiones éticas lo que podría frenar la regresión de la capacidad de juicio moral de los estudiantes de medicina" (Delgado-Marroquín et al., 2014, p. 57). En este orden de ideas, el trabajo en pequeños grupos para resolver complejos 
dilemas éticos relacionados con la práctica profesional ofrece un campo ideal para observar los marcos que emplea cada estudiante para la argumentación y resolución de casos, e identificar estudiantes en riesgo de conductas que faltan a la integridad académica (Henning et al., 2013).

Resultaría entonces definitivo para el desarrollo moral de un estudiante el entorno y el contexto cultural que lo rodea. Convertirse en un profesional de la medicina, tal como la sociedad lo reclama, no depende únicamente de egresar de un prestigioso programa, sino también de los pares con los que se ha compartido el proceso de formación. Entonces, se convierte en una prioridad para las instituciones con programas de Medicina de alta calidad el trabajar en la cultura de la integridad académica, así como el empoderamiento de los estudiantes en este proceso como instancia de autorregulación.

En relación con las faltas a la conducta académica, son diversas las argumentaciones que se encuentran al confrontar a los estudiantes implicados, las cuales revelan el grado de desarrollo del razonamiento moral en el que se encuentran. Algunas de estas son las siguientes:

Se falsean incapacidades, debido a que esta es la única excusa que es aceptada, por lo tanto "los estudiantes son forzados a mentir"; si la evaluación no es importante y tiene poco peso, no está mal copiar (Ghias et al., 2014). Ayudar a un amigo en la copia no se considera una falta (Brockbank, David y Patel,
2011). Los estudiantes que faltan a la integridad académica racionalizan con frecuencia su comportamiento y niegan las implicaciones negativas de esta conducta para terceros (Staats et al., 2009).

No es de extrañar que cuando se les pide ponderar las faltas a la integridad académica, al público en general, a los médicos graduados y a los estudiantes, estos últimos consideran que faltas como inasistencia, mentiras en la examinación de los pacientes y falsificación de datos en investigación no son importantes y deben ser condonables (Brockbank, David y Patel, 2011).

Las actitudes de los estudiantes revelan la etapa del desarrollo moral en la que se encuentran, acorde con la escala de Kohlberg (Pérez-Olmos y Dussán-Buitrago, 2009):

- Selectivamente se falta o no a la integridad académica, dependiendo de que exista o no la posibilidad de castigo ("nivel I preconvencional, etapa I de moralidad heterónoma").

- A pesar de que se observan faltas a la integridad académica, no se denuncian, "se vive y se deja vivir" ("nivel I preconvencional, etapa 2, de individualismo, propósito instrumental e intercambio").

- Dejar copiar a un amigo es justo y refleja un acto de lealtad ("nivel II convencional, etapa 3 expectativas interpersonales mutuas, de relaciones y de conformidad interpersonal"). 
- Los estudiantes que no faltan a la integridad académica, a pesar de que sea una práctica socialmente aceptada, porque consideran que no es justo, que las notas obtenidas de esta forma no tienen valor y que tienen una responsabilidad social sobre su formación, pueden corresponder a un nivel III postconvencional.

Para resumir, es necesario evaluar el desarrollo moral al ingreso y que este sea incorporado dentro de los criterios de admisión. Por otra parte, se debe promover un ethos dentro de cada cohorte, en el programa y en los espacios clínicos y comunitarios, que propenda hacia la integridad académica, en el cual se detecten, evalúen y sancionen, de forma oportuna y justa, las desviaciones y se incentiven las buenas prácticas. Es necesario evaluar los progresos en el desarrollo moral para identificar e intervenir, mediante consejería, revisión por pares o modelos de mentoría, aquellos estudiantes que no adquieren las competencias esperadas.

\section{Motivaciones para el aprendizaje autónomo}

¿Cómo lograr que los estudiantes aprendan más allá de lo requerido para sus evaluaciones y que puedan enfrentar esta parte de su proceso formativo sin recurrir al engaño?, ¿cómo lograr que se encaminen hacia una ética de máximos que los incentive a esforzarse más allá de la adquisición de una competencia básica?
Las motivaciones que tienen los estudiantes para estudiar son externas e internas; no obstante, las últimas son aquellas que fomentan la excelencia. La motivación autónoma, que nace de un interés genuino o un valor personal, es más efectiva que la motivación controlada (la cual depende de factores externos como la presión de maestros, padres, las evaluaciones, la culpa, la vergüenza o los premios), y se asocia con un mejor desempeño académico, el aprendizaje profundo, menor agotamiento y la capacidad para actualizarse a lo largo de la vida. Esta debe ser estimulada mediante estrategias pedagógicas que le permitan al estudiante percibir que tiene la posibilidad de elegir. Esto se logra con la enseñanza en grupos pequeños, el aprendizaje basado en problemas y el aumento en espiral de responsabilidades, así como con un adecuado feed back sobre sus resultados (Kusurkar y Croiset, 2015).

El aprendizaje autónomo requiere también retos, los cuales deben ser óptimos (ni muy fáciles, ni muy difíciles), lo cual varía de un estudiante a otro y del momento de la formación (Kusurkar y Croiset, 2015). No debe confundirse autonomía con independencia o anarquía, debido a que el proceso de aprendizaje demanda supervisión (Schumacher, Bria y Frohna, 2013). Este hecho solicita que, además de la evaluación del cumplimiento de los Resultados de Aprendizaje Esperados, se identifiquen aquellos estudiantes que requieren desafíos intelectuales o emocionales adicionales, los 
cuales se encuentran, por ejemplo, en actividades relacionadas con la investigación o el liderazgo académico (consejos estudiantiles, tutoría a otros estudiantes).

Esto nos lleva a preguntarnos: ¿será necesario seleccionar a los aspirantes a los programas de Medicina teniendo en cuenta sus motivaciones y valores o en sus puntajes de razonamiento moral?, ¿debemos seguir seleccionando a los aspirantes con base en el buen rendimiento académico en el colegio o en las pruebas de Estado, más aun cuando la falta de honestidad académica inicia en la educación escolar?

Sin lugar a dudas también es necesario intervenir las distintas dimensiones del currículo oculto negativo en los espacios de las ciencias básicas y biomédicas y de las ciencias clínicas, debido a que nada hacemos con seleccionar personas proclives al altruismo y con un adecuado desarrollo de juicio moral, cuando el entorno los transforma adversamente.

\section{¿Cuáles son los efectos de las prác- ticas deshonestas?}

Los efectos de este tipo de prácticas inciden sobre distintos grupos de personas: los estudiantes, los docentes y responsables de los procesos disciplinarios, las instituciones y el sistema educativo, la profesión médica y la sociedad en su conjunto.

Para el estudiante, un error cometido durante el proceso de formación puede, en el mejor de los escenarios, convertirse en un antecedente en su historia académica, lo que disminuye sustancialmente su posibilidad de ingresar exitosamente a los competidos posgrados clínico-quirúrgicos o al espacio laboral. En el peor de los casos, recibir sanciones que incluyen la pérdida de la asignatura, la amonestación privada, la suspensión o la expulsión definitiva y que incluso, en casos graves o reincidencia, pueden generar responsabilidad jurídica de tipo penal o civil.

Por otra parte, el estudiante que ha fundamentado su proceso educativo en el engaño no contará con las competencias para practicar la profesión de manera segura, lo cual se convertirá en un riesgo ético y jurídico en su ejercicio profesional futuro. Tampoco adquirirá las habilidades para el aprendizaje a lo largo de la vida y se desactualizará rápidamente. Finalmente, se afectará su desarrollo como persona, la posibilidad de ser creativo, así como la adquisición de los valores requeridos para desempeñarse como ciudadano y profesional, lo cual influirá negativamente en todas las esferas de su vida (Ghias et al., 2014).

El trabajo en grupo dentro del proceso de formación médica tiene como fines, entre otros, el desarrollo de valores como la lealtad y la confianza entre los miembros del grupo, y el desarrollo de la autorregulación y la regulación entre pares (Hendry, Hyde y Davy, 2005).

Las conductas académicas deshonestas inciden en el desempeño de los otros estudiantes, alteran el clima de confianza entre colegas que debe existir en el 
interior de un programa de Medicina, arrastran negativamente el desempeño académico del grupo e impiden o dilatan el desarrollo moral de todos los miembros de una cohorte. Finalmente, los códigos de silencio que se construyen desde la vida universitaria impiden que aquellos que observan estas prácticas en sus compañeros, y no las denuncian, cumplan en el futuro con la obligación profesional de actuar, en el contexto de la autorregulación, cuando las desviaciones en la práctica profesional de un colega colocan en riesgo la integridad de un paciente (Goldie, Schwartz, McConnachie y Morrison, 2003).

Para las instituciones universitarias es un desperdicio tener que emplear recursos que deberían ser destinados para otras prioridades de la educación, en detectar y sancionar estas prácticas. Por otro lado, cuando existe la percepción de que la falta de integridad académica se ha generalizado, los docentes y las personas responsables de los procesos disciplinarios pueden excederse en las acciones de detección y corrección, e incluso polarizar sus decisiones y hacerlas más severas. Si los índices de faltas a la integridad académica son altos, la universidad incumple su promesa de servicio a la sociedad de formar ciudadanos y profesionales.

Para la sociedad, lamentablemente, el engaño que se da en el escenario académico se reitera en el laboral y tiene la potencialidad de hacer daño a las personas (Rujoiu y Rujoiu, 2014). No es de extrañar que cada vez sean más frecuentes las quejas sobre la falta de integridad de los profesionales de la medicina, en su relación con los pacientes, colegas, aseguradores, agentes de control e industria farmacéutica, que se hacen evidentes en fallas en la atención, conducta sexual inapropiada, abuso de sustancias, problemas en la prescripción y fraude en seguros (Garzón, 2000). Incluso, la mala conducta durante el proceso de educación médica se considera un factor de riesgo para las desviaciones en la conducta profesional (Turrens, Staik, Gilbert, Small y Burling, 2004).

La sociedad espera de los médicos la excelencia ética en su comportamiento, con respeto a valores como la verdad, la integridad, la vida, la salud, la intimidad, entre otros. Cuando un miembro de la profesión médica es incapaz de obrar profesionalmente, la sociedad desconfía de todos sus miembros y responde con regulación, lo que limita los privilegios de ejercicio autónomo y autorregulado (Escobar-Melo et al., 2000).

Además, dado que el acceso a la educación médica está limitado por sus altos costos, es una injusticia que las pocas personas que logran ingresar y culminar sus estudios no posean las competencias requeridas para satisfacer las necesidades de salud de las personas y las comunidades.

\section{¿Cómo contrarrestar la falta de integridad académica?}

Debido a que esta problemática es el resultado de diferentes causas, las 
estrategias que se proponen para enfrentarla deben ser diversas (Kusnoor y Falik, 2013) y se agrupan en las de carácter preventivo, de detección y sancionatorias (Aunión, 2013).

Algunos plantean la orientación y el entrenamiento de la comunidad académica en aspectos de integridad académica, incluida la política de la institución, la implementación de un proceso que se encargue de este tipo de violaciones, lograr que el estudiante se apropie del concepto de integridad académica y se empodere en su proceso educativo, mantener el diálogo con los afectados por la deshonestidad académica, refinar los aspectos éticos del currículo, monitorear los esfuerzos, documentar y comunicar los resultados (Rujoiu y Rujoiu, 2014).

Dentro de las medidas preventivas se encuentran las siguientes:

- Creación de oficinas de integridad académica, donde pueda promoverse una cultura de la honestidad y se lideren los distintos procesos asociados. Se recomienda que un grupo conformado por las autoridades del programa de Medicina, los docentes y los estudiantes diseñen y divulguen una política clara sobre la integridad académica. Esta debe incluir la definición y los ejemplos de este tipo de conducta, el proceso de denuncia o de investigación, los derechos y responsabilidades de los estudiantes, docentes y responsables de la gestión del programa, las sanciones asociadas y la conformación y el funcionamiento de los comités disciplinarios, entre otros elementos (Gitanjali, 2004).

- Existencia de códigos de honor o de conducta dentro de las universidades. La literatura indica que aquellas universidades en las que se cuenta con estos instrumentos, el porcentaje de copia es menor. Esto se debe a que cuando existe claridad sobre aquello que es correcto o incorrecto, es más difícil justificar la práctica deshonesta. Sin embargo, no cumplen con su fin cuando los valores que se enuncian en ellas no son internalizados por los estudiantes o si no se explica claramente qué hacer cuando se observan estas prácticas. Se señala que se hacen más necesarios en la medida en que el contacto con los pacientes es más temprano (Anderson y Pickering, 2010).

- Uso de declaraciones de honestidad académica al momento de la matrícula, en las que los estudiantes se comprometen por escrito a no copiar. Estas se deben acompañar de la información sobre políticas de integridad académica de la universidad. Este tipo de declaraciones también se escriben antes de cada prueba o entrega de trabajo. En todos los syllabus se debe incluir las consecuencias de faltar a la integridad académica (Scanlan, 2006).

- Límite al número de estudiantes por clase, de modo que permita un 
mayor control y personalización del proceso formativo (Ledesma, 2011).

- Concentrar el interés de la Facultad o Escuela en el proceso de aprendizaje y no en el de evaluación. Se debe fomentar el aprendizaje autónomo, con supervisión y personalización de los retos (Rennie y Crosby, 2001).

- Enseñanza de los aspectos éticos de la integridad académica y ética profesional en los currículos de medicina de forma temprana y reforzarlos a lo largo del currículo (Scanlan, 2006).

- Mejora en la formación ética, tanto desde el currículo formal, como en el oculto, con fomento de los modelos de rol (Ghias et al, 2014).

- Disminuir las posibilidades de copia a través de diversas estrategias como son, entre otras: uso de pruebas generadas aleatoriamente por computador, o test alternados, lugares asignados al azar para la evaluación, y la exclusión de dispositivos electrónicos durante las pruebas (Scanlan, 2006).

- Procurar no repetir preguntas que se han hecho en el pasado y diseñar pruebas que no favorezcan la copia (como es el caso de las pruebas de selección múltiple). (Gitanjali, 2004).

- Intervención de los factores que se relacionan con el estrés y el síndrome de agotamiento (Reed, Shanafelt, Satele et al., 2011). Esto implica cambios en los modelos de poder jerárquico, tan usual en los escenarios médicos, disminuir la sobrecarga de créditos, proteger tiempos para el descanso y las actividades personales, entre otras.

Dentro de las medidas de detección están:

- Contar con la estricta supervisión de las pruebas, las cuales no deben ser delegadas a terceros que no entiendan la importancia de este proceso (Gitanjali, 2004).

- Evaluación por pares y los procesos de consejería (Kusnoor y Falik, 2013).

- Incluir en los códigos de honor de los estudiantes el deber de reporte cuando tengan conocimiento de este tipo de prácticas, reforzando su continuidad como deber profesional futuro cuando se coloca en peligro el bienestar de un paciente. Esto incluye dar mayores responsabilidades a los estudiantes sobre la vigilancia de la integridad académica (Scanlan, 2006). Cuando un estudiante denuncia, se debe proteger su anonimato.

- Uso de software para detectar plagio, como Turnitin.com, MyDropBox.com, Essay Verification Engine, Plagiarism Finder, CopyCatch, WCopyfind y Glatt Plagiarism Screening program (Scanlan, 2006).

En lo que respecta a las medidas sancionatorias, un estudio afirma que un $90 \%$ de los estudiantes considera que pueden copiar y no serán detectados, ni sancionados (Scanlan, 2006). En este 
orden de ideas, algunos autores apuntan a la necesidad de contar con políticas de cero tolerancia ante cualquier falta a la honestidad académica (Ledesma, 2011). Una institución, una escuela o facultad, una comunidad académica que se muestra indulgente con este tipo de prácticas, implícitamente envía el mensaje de que se deben tolerar las desviaciones en la práctica profesional (Gitanjali, 2004).

Dentro de las sanciones se propone la pérdida de la asignatura, la suspensión e incluso la expulsión, en razón de la gravedad de la falta y su carácter repetitivo. Las personas que son sancionadas pueden perder otro tipo de privilegios, como aspirar a becas, grupos estudiantiles, representar a la universidad, entre otros. Por otra parte, este tipo de faltas se incluyen en los expedientes académicos y repercuten en procesos académicos de posgrado (Scanlan, 2006).

\section{Conclusiones}

Las Universidades y su comunidad académica son responsables ante la sociedad de la formación ciudadana y profesional de sus egresados. La estrategia que se emplee para cumplir con este fin debe ser multifactorial y apuntar a contrarrestar los factores de riesgo e incentivar positivamente los protectores. Esta debe ser transversal a los procesos de admisión, permanencia y egreso del programa.

Se debe propender hacia una cultura de la integridad académica, donde se sancionen socialmente los comporta- mientos deshonestos. Esta se basa en una política que tiene que ser construida entre todos los miembros de la comunidad académica y la cual es importante que sea socializada. Se debe contar con mecanismos efectivos de prevención, detección y sanción.

Les corresponde a los docentes y directivos colocar dentro de sus prioridades la garantía de un ambiente formativo donde prime la integridad académica. Este compromiso se expresa en esfuerzos por mejorar los procesos de admisión, permanencia, evaluación y control sobre los resultados. Asimismo, debe otorgarse valor, no solo a la excelencia académica en términos de resultados académicos, sino también a la forma como esta se ha alcanzado con un enfoque centrado en el proceso de formación.

Es primordial que la comunidad académica esté atenta al uso de las tecnologías de la comunicación y la información con fines deshonestos en el ámbito académico, y responder de forma creativa y anticipada.

El desarrollo moral de los estudiantes debe ser un foco de la enseñanza de la medicina. Con este fin, es necesario determinar el estadio moral al ingreso (incluso como parte de los criterios de selección) y su progreso durante el proceso formativo, para detectar aquellos estudiantes con problemas en esta área profesional, ofrecerles apoyo, consejería y acompañamiento.

El aprendizaje autónomo ha de ser supervisado e individualizado; de igual 
forma, tiene que delegarse responsabilidades a los estudiantes de manera progresiva. No basta con cumplir con los resultados esperados de aprendizaje; se deben propiciar actividades que desafíen permanentemente a los estudiantes en la medida de sus capacidades. El contacto temprano con los pacientes y los ámbitos hospitalarios nos obliga a estar atentos a cambios más tempranos en las escalas de razonamiento moral.

\section{Referencias}

Anderson, L.C. y Pickering, N.J. (2010). The student code: ethical and professional expectations of medical students at the University of Otago. The New Zealand Medical Journal, 123(1318), 43-49.

Anderson, R.E. y Obenshain, S.S. (1994). Cheating by students: findings, reflections, and remedies. Academic Medicine, 69(5), 323-32.

Aunión, J.A. (2013). No copiaré, no copiaré, no copiaré, no copiaré. El País. Recuperado de https:// elpais.com/sociedad/2013/09/18/ actualidad/1379539445_080241.html

Baird Jr., J.S. (1980). Current trends in college cheating. Psychology in the Schools, 17(4), 515-522.

Baldwin Jr, D.C., Daugherty, S.R., Rowley, B.D. y Schwarz, M.D. (1996). Cheating in medical school: A survey of secondyear students at 31 schools. Academic Medicine, 71(3), 267-73.

Batane, T. (2010). Turning to Turnitin to Fight Plagiarism among University Students. Educational Technology \& Society, 13(2), 1-12.
Bates, I.P., Davies, J.G., Murphy, C. y Bone, A. (2005). A multi-faculty exploration of academic dishonesty. Pharmacy Education, 5(1), 69-76.

Bermeo J.L., Castaño-Castrillón, J.J., LópezRomán, A., Téllez, D.C. y Toro-Chica, S. (2016). Abuso académico a estudiantes de pregrado por parte de docentes de los programas de Medicina de Manizales, Colombia. Revista de la Facultad de Medicina, 64(1), 9-19.

Bjorklund, M. y Wenestam, C.G. (22-25 de septiembre de 1999). Academic cheating: frequency, methods, and causes. En European Conference on Educational Research. Lahti, Finland.

Bonke, B. (2006). Unprofessional or problematic behaviour of medical students outside the learning environment. Medical Teacher, 28(5), 440-442.

Brockbank, S., David, T.J. y Patel, L. (2011). Unprofessional behaviour in medical students: a questionnaire-based pilot study comparing perceptions of the public with medical students and doctors. Medical Teacher, 33(9), e501-508.

Christakis, D.A. y Feudtner, C. (1993). Ethics in a short white coat: the ethical dilemmas that medical students confront. Academic Medicine, 68(4), 249-54.

Delgado-Marroquín, M., Altisent, R., Buil, B., Muñoz, P. et al. (2014). Análisis de la maduración del juicio moral en el proceso de aprendizaje de la bioética. FEM: Revista de la Fundación Educación Médica, 17(1), 55-62.

Davis, S.F. y Ludvigson, H.W. (1995). Additional data on academic dishonesty 
and a proposal for remediation. Teaching of Psychology, 22(2), 119-21.

De Oliveira Vidal, E.I, Silva, V., Santos, M.F. et al. (2015). Why Medical Schools Are Tolerant of Unethical Behavior. Annals of Family Medicine, 13(2), 176-80.

Dyrbye, L.N., Massie, F.S. Jr, Eacker, A. et al. (2010). Relationship between burnout and professional conduct and attitudes among US medical students. JAMA, 304(11), 1173-80.

Escobar-Melo, H., Díaz, E; Páramo, L.C., Suárez, F. y León, C. (2010). Caracterización del razonamiento moral en la práctica clínica en relación con el proceso de formación médica en una facultad de medicina, categoría altruismo y dignidad, beneficencia, confidencialidad y deber de cuidado. Revista Colombiana de Bioética, 5(1), 34-56.

Franklyn-Stokes, A. y Newstead, S.E. (1995). Undergraduate cheating: who does what and why? Studies in Higher Education, 20(2), 159-72.

Freidson, E. (2001). La teoría de las profesiones: Estado del arte. Perfiles educativos, 23(93), 28-43.

Freidson, E. (1988). Profession of medicine: a study of the sociology of applied knowledge. Chicago: University of Chicago Press.

García, E. y Manchado, B. (1998). Un modelo econométrico del fraude académico en una universidad española. Universidad Complutense de Madrid. Recuperado de http://eprints.ucm.es/6672/

Garzón, F. (2000). Bioética: manual interactivo. Bogotá: 3R Editores.

Gehring, D.D. y Pavela, G. (1994). Issues and perspectives on academic integrity. (2da. Ed.). Washington, D. C.: National Association of Student Personnel Administrators.

Ghias, K., Lakho, G.R, Asim, H., Azam, I.S. y Saeed, S.A. (2014). Self-reported attitudes and behaviours of medical students in Pakistan regarding academic misconduct: a cross-sectional study. BMC Medical Ethics, 15, 43.

Gitanjali, B. (2004). Academic dishonesty in Indian medical colleges. Journal of Postgraduate Medicine, 50(4), 281284.

Goldie, J., Schwartz, L., McConnachie, A. y Morrison, J. (2003). Students' attitudes and potential behaviour with regard to whistle blowing as they pass through a modern medical curriculum. Medical Education, 37(4), 368-375.

González, B. (2014). El pinganillo: la microchuleta que triunfa para copiar en los exámenes. El Mundo. Recuperado de http://www.elmundo.es/andaluci a/2014/03/16/5322e47322601dfd4e 8b4579.html

Hafeez, K., Khan, M.L.Z., Jawaid, M. y Haroon, S. (2013). Academic misconduct among students in medical colleges of Karachi, Pakistan. Pakistan Journal of Medical Sciences, 29(3), 699-702.

Hendry, G.D., Hyde, S.J. y Davy P. (2005). Independent student study groups. Medical Education, 39(7), 672-679.

Henning, M.A., Ram, S., Malpas, P., Shulruf, B. et al. (2013). Academic dishonesty and ethical reasoning: Pharmacy and medical school students in New Zealand. Medical Teacher, 35(6), e1211-e1217.

Hren, D., Marušić, M. y Marušić, A. (2011). Regression of Moral Reasoning during 
Medical Education: Combined Design Study to Evaluate the Effect of Clinical Study Years. PLoS One, 6(3), e17406.

Kleiner, C. y Lord, M. (1999). The cheating game: "Everyone's doing it", from grade school to graduate school. US News \& World Report, 55-66.

Kulac, E., Sezik, M., Asci, H. y Doguc, D.K. (2013). Medical students' participation in and perception of unprofessional behaviors: comparison of preclinical and clinical phases. Advances in Physiology Education, 37(4), 298-302.

Kusnoor, A.V. y Falik, R. (2013). Cheating in Medical School: The Unacknowledged Ailment. Southern Medical Journal, 106(8), 479-483.

Kusurkar, R.A. y Croiset, G. (2015). Autonomy support for autonomous motivation in medical education. Medical Education Online, 20, 27951.

Lázaro, J. y Gracia D. (2006). La relación médico-enfermo a través de la historia. Anales Sis San Navarra, 29(3), 7-17.

Ledesma, R.G. (2011). Academic Dishonesty among Undergraduate Students in a Korean University. Research in World Economy, 2(2), 26.

McCabe, D.L. y Trevino, L.K. (1997). Individual and contextual influences on academic dishonesty: a multicampus investigation. Research in Higher Education, 38(3), 379-96.

McCabe, D.L., Trevino, L.K., Butterfield, K.D. (2001). Cheating in academic institutions: a decade of research. Ethics Behavior, 11(3), 219-232.

MacDougall, M. (2008). Ten tips for promoting autonomous learning and effective engagement in the teaching of statistics to undergraduate medical students involved in short-term research projects. Journal of Applied Quantitative Methods, 3(3), 223-240.

Neumann, M., Edelhäuser, F., Tauschel, D. et al. (2011). Empathy decline and its reasons: a systematic review of studies with medical students and residents. Academic Medicine, 86(8), 996-1009.

Newstead, S.E., Franklyn-Stokes, A. y Armstead, P. (1996). Individual differences in student cheating. Journal of Educational Psychology, 88(2), 229-241.

Ordoñez, C.O., Mejía, J.F. y Castellanos, S. (2006). Percepciones estudiantiles sobre el fraude académico: hallazgos y reflexiones pedagógicas. Revista de Estudios Sociales, 23, 37-44.

Patenaude, T., Niyonsenga, T. y Fafard, D. (2003). Changes in the components of moral reasoning during students' medical education: a pilot study. Medical Education, 37(9), 822-829.

Pérez-Olmos, I. y Dussán-Buitrago, M.M. (2009). Validación de la prueba Defining Issues Test con estudiantes de Medicina en la Universidad del Rosario, en Colombia. Revista Electrónica de Investigación Educativa, 11(1), 1-13.

Pupovac, V., Bilic-Zulle, L., Petrovecki, M. (2008). On academic plagiarism in Europe. An analytic approach based on four studies. En R. Comas, R. y J. Sureda (Coords.). Academic cyberplagiarism. Recuperado de http://www.uoc.edu/ digithum/10/dt/eng/pupovac_biliczulle_petrovecki.pdf

Quintero, G.A. (Ed.). (2014). Actividades integradoras del aprendizaje por sistemas, 
AIAS. Manual para la facilitación en el aula de pequeños grupo. Bogotá: Editorial del Rosario.

Rodríguez, J.A. (1981). Estructura de la profesión médica española. Reis: Revista Española de Investigaciones Sociológicas, 14, 95-112.

Rennie, S.C. y Rudland, J.R. (2003). Differences in medical students' attitudes to academic misconduct and reported behavior across the years - a questionnaire study. Journal of Medical Ethics, 29(2), 97-102.

Reed, D.A., Shanafelt, T.D., Satele, D.W. et al. (2011). Relationship of pass/fail grading and curriculum structure with well-being among preclinical medical students: a multi-institutional study. Academic Medicine, 86(11), 1367-1373.

Rennie, S.C. y Crosby, J.R. (2001). Are "tomorrow's doctors" honest? Questionnaire study exploring medical students' attitudes and reported behaviour on academic misconduct. BMJ Open, 322(7281), 274-275.

Rennie, S.C. y Crosby, J.R. (2002). Students' perceptions of whistle blowing: implications for self-regulation. A questionnaire and focus group survey. Medical Educcation, 36(2), 173-179.

Rujoiu, O. y Rujoiu, V. (2014). Academic dishonesty and workplace dishonesty. An overview. En 8th International Management Conference "management challenges for sustainable development". Bucharest, Romania.

Scanlan, C.L. (2006). Strategies to Promote a Climate of Academic Integrity and
Minimize Student Cheating and Plagiarism. Journal of Allied Health, 35(3), 179-185.

Schumacher, D.J., Bria, C. y Frohna, J.G. (2013). The quest toward unsupervised practice: promoting autonomy, not independence. JAMA, 310(24),26132614.

Sheriff, D.S., Sheriff, S.O. y Manopriya, M. (2000). Higher education on a pedestal of academic dishonesty. Eubios Journal of Asian and International Bioethics, 10(1), 6-8.

Smith, R. (2000). Cheating at medical school. BMJ Open, 321(7258), 398.

Staats, S., Hupp, J.M., Wallace, H. y Gresley, J. (2009). Heroes don't cheat: An examination of academic dishonesty and students' views on why professors don't report cheating. Ethics \& Behavior, 19(3), 171-183.

Turrens, J.F., Staik, I.M., Gilbert, D.K., Small, W.C. y Burling, J.W. (2001). Undergraduate academic cheating as a risk factor for future professional misconduct. En N. H. Steneck y M. D. Scheetz (Eds.), Proceedings of the First ORI Research Conference on Research Integrity (pp. 19-20). Maryland: Office of Research Integrity.

Universidad de Edimburgo. (2016). Academic misconduct. Recuperado de http:// www.ed.ac.uk/academic-services/staff/ discipline/academic-misconduct

Vengoechea, J., Moreno, S. y Ruíz, A. (2008). Misconduct in Medical Students. Developing World Bioethics, 8(3), 219225. 